\title{
Comparison of Oral Sulfate Solution and Polyethylene Glycol Plus Ascorbic Acid on the Efficacy of Bowel Preparation
}

\author{
Ji Hyung Nam*, Seok Bo Hong*, Yun Jeong Lim, Seongju Lee, Hyoun Woo Kang, Jae Hak Kim and Jin Ho Lee \\ Department of Internal Medicine, Dongguk University Ilsan Hospital, Goyang, Korea
}

Background/Aims: The quality of bowel preparation is one of the quality indicators for colonoscopy. The aim of this study was to compare the efficacy of oral sulfate solution (OSS) and polyethylene glycol plus ascorbic acid (PEG-AA) for bowel preparation.

Methods: The study involved 167 patients who underwent diagnostic colonoscopies. Inadequate bowel preparation was defined as any score of $\leq 1$ in each colon section based on the Boston Bowel Preparation Scale. Multivariate logistic regression was used to compare the efficacy of OSS and PEG-AA. Subgroup analyses were performed based on patient characteristics.

Results: Overall, $106(63.5 \%)$ patients received OSS, and 61 (36.5\%) patients received PEG-AA. The rate of inadequate bowel preparation was $12.3 \%$ in patients receiving OSS and $32.8 \%$ in patients receiving PEG-AA $(p=0.001)$. OSS (odds ratio [OR] = 0.26; $p=0.003)$ and morning examination $(\mathrm{OR}=0.11 ; p=0.038)$ were significantly associated with efficient bowel preparation. The efficacy of OSS compared with PEG-AA was only significant in patients $\geq 50$ years of age vs. $<50$ years of age $(\mathrm{OR}=0.13 ; p=0.001 \mathrm{vs}$. OR=0.96; $p=0.959)$ and female vs. male patients $(\mathrm{OR}=0.06 ; p=0.002$ vs. $\mathrm{OR}=0.58 ; p=0.339)$.

Conclusions: OSS was significantly more efficient for bowel preparation than PEG-AA, especially in patients $\geq 50$ years of age and female patients. Morning examination led to a good quality of bowel preparation, irrespective of the preparation regimen. Clin Endosc 2020;53:568-574

Key Words: Bowel preparation; Colonoscopy; Oral sulfate solution; Polyethylene glycol

\section{INTRODUCTION}

Colonoscopy allows the early diagnosis for colorectal cancer (CRC), as well as the treatment of precancerous lesions, which can help prevent CRC. ${ }^{1,2}$ Qualified colonoscopy is enabled by meticulous observation of the entire colonic mucosa, which is subject to adequate bowel preparation. Inadequate bowel preparation accounts for $25 \%$ of failed colonoscopies. ${ }^{3}$ In addition to the cecal intubation rate and adenoma detection rate

Received: November 5, 2019 Revised: March 20, 2020

Accepted: March 23, 2020

Correspondence: Yun Jeong Lim

Division of Gastroenterology, Department of Internal Medicine, Dongguk University Ilsan Hospital, 27 Dongguk-ro, Ilsandong-gu, Goyang 10326, Korea Tel: +82-31-961-7133, Fax: +82-31-961-9339, E-mail: drlimyj@gmail.com ORCID: https://orcid.org/0000-0002-3279-332X

*These authors contributed equally to this study.

(c) This is an Open Access article distributed under the terms of the Creative Commons Attribution Non-Commercial License (http://creativecommons.org/ licenses/by-nc/3.0) which permits unrestricted non-commercial use, distribution, and reproduction in any medium, provided the original work is properly cited.
(ADR), the quality of bowel preparation is a known quality indicator for colonoscopy. ${ }^{4,5}$ Inadequate bowel preparation can reduce the $\mathrm{ADR}$, and increase procedure time and the resultant patient discomfort, the risk of complications, and the costs associated with shortened surveillance intervals. ${ }^{6-9}$

The choice of an effective and tolerable preparation regimen is of utmost importance. Several Food and Drug Administration (FDA)-approved formulations for bowel cleansing are currently used, including polyethylene glycol (PEG)-electrolyte lavage solution, for example, 4 L PEG and 2 L PEG plus ascorbic acid (PEG-AA); oral sulfate solution (OSS); sodium picosulfate; and sodium phosphate in limited use. ${ }^{2,3,10}$ These bowel preparation agents have been selected based on efficacy, safety, and tolerability. The standard volume (4 L) of PEG is safe and has been used as the most effective formulation, but there is low compliance because of the large intake volume. ${ }^{11}$ Low-volume (2 L) PEG-AA is more tolerable than the standard volume PEG and has comparable safety. ${ }^{12,13}$ The decreased volumes needed for OSS and sodium picosulfate (less than $1 \mathrm{~L}$ ) have increased their popularity. Although these formulations 
are proven to be effective and safe, distinct features, such as taste, medication guides, and total dosage, can influence tolerability and compliance, depending on the characteristics of the individual. Concurrently, several randomized clinical trials have shown that OSS has comparable or better outcomes than PEG formulations and has acceptable safety. ${ }^{14-16}$ Thus, this observational study aimed to compare the efficacy of OSS and PEG-AA and to identify the patient characteristics that were favorable to certain formulations.

\section{MATERIALS AND METHODS}

\section{Study design}

This cross-sectional study was conducted on consecutive patients who underwent diagnostic colonoscopies at Dongguk University Ilsan Hospital between May 2016 and August 2016. The exclusion criteria were insufficient information about the bowel cleansing quality, therapeutic colonoscopy for alleged colorectal neoplasms, and previous history of colorectal resection. This study was approved by the Institutional Review Board (DUIH 2017-11-013-003).

All patients were examined by experienced endoscopists using a video colonoscope (Olympus CF-H260 or CF-Q260; Olympus Optical Co., Ltd., Tokyo, Japan). Intravenous midazolam was administered to the patients who wanted sedative endoscopies. The dose was determined according to a unified protocol based on the subject's age and weight. Meperidine was applied routinely as an analgesic.

\section{Bowel preparation}

Bowel cleansing was achieved with either OSS (Innofree; MH Healthcare, Seoul, Korea) or PEG-AA (Coolprep; Taejoon Pharm. Co., Seoul, Korea). OSS contained 17.5 g sodium sulfate, $3.13 \mathrm{~g}$ potassium sulfate, $1.6 \mathrm{~g}$ magnesium sulfate, and flavoring agents in an aqueous liquid form supplied in a $177 \mathrm{~mL}$ bottle. The water was filled up to the $473 \mathrm{~mL}$ line marked on the bottle. The patients were instructed to take the diluted OSS with an additional $1 \mathrm{~L}$ of water at the first dose and to repeat the same preparation with the second dose. The OSS formulation was administered as a split-dose at 12-h intervals for all patients without exception (5 pm on the day before the colonoscopy and $5 \mathrm{am}$ on the day of the procedure for morning colonoscopies; or $7 \mathrm{pm}$ on the day before the colonoscopy and $7 \mathrm{am}$ on the day of the procedure for afternoon colonoscopies). The PEG-AA contained 100 g PEG, 1 g potassium chloride, $2.69 \mathrm{~g}$ sodium chloride, $7.5 \mathrm{~g}$ sodium sulfate, $4.7 \mathrm{~g}$ ascorbic acid, and $5.9 \mathrm{~g}$ sodium ascorbate per liter. The patients were instructed to take 1 L PEG-AA solution with an additional $500 \mathrm{~mL}$ water at the first dose and to repeat the same preparation with the second dose. For the PEG-AA formulation, both split-dose and single-dose methods were available. For afternoon colonoscopies, 2 L PEG was taken on the day of the examination at 3-hour intervals $(1 \mathrm{~L}$ each at 5 am and $8 \mathrm{am}$ ) for the single-dose method. For morning colonoscopies, $1 \mathrm{~L}$ was taken on the day before the examination $(8$ $\mathrm{pm}$ ) and another $1 \mathrm{~L}$ was taken on the day of the procedure (5 $\mathrm{am})$ in the split-dose method.

The quality of bowel cleansing was assessed by physicians blinded to the bowel preparation regimens using the Boston Bowel Preparation Scale (BBPS). ${ }^{7}$ The BBPS assesses the cleanliness of the right colon (cecum and ascending colon), transverse colon (hepatic flexure, transverse, and splenic flexure), and left colon (descending, sigmoid colon, and rectum) (Table 1). Each colon section is individually rated with scores from 0 to 3, yielding total scores ranging from 0 (completely unprepared) to 9 (perfectly prepared). In the study, the quality of bowel preparation was considered adequate when all scores in each colon section were 2 or 3 . If any of the colon sections scored 1 or 0 , the preparation was considered inadequate (Fig. 1).

Table 1. Boston Bowel Preparation Scale

\begin{tabular}{|c|c|c|}
\hline & Score & Definition \\
\hline \multirow{4}{*}{$\begin{array}{l}\text { Section score for each colon } \\
\text { section (right, transverse, and } \\
\text { left colon) }{ }^{\text {a) }}\end{array}$} & 0 & Unprepared colon segment with mucosa not seen because of solid stool that cannot be cleared \\
\hline & 1 & $\begin{array}{l}\text { Portion of mucosa of the colon segment seen, but other areas of the colon segment are not well } \\
\text { seen because of staining, residual stool, and/or opaque liquid }\end{array}$ \\
\hline & 2 & $\begin{array}{l}\text { Minor amount of residual staining, small fragments of stool, and/or opaque liquid, but mucosa } \\
\text { of colon segment is seen well }\end{array}$ \\
\hline & 3 & $\begin{array}{l}\text { Entire mucosa of colon segment seen well, with no residual staining, small fragments of stool, } \\
\text { or opaque liquid }\end{array}$ \\
\hline Sum of scores & $0-9$ & From 0 (non-prepared colon) to 9 (perfectly clean colon) \\
\hline
\end{tabular}

${ }^{a}$ Right, cecum to the ascending; transverse, hepatic to the splenic flexure; Left, descending to the rectum. 
C clinical endoscopy

OSS (inadequate)

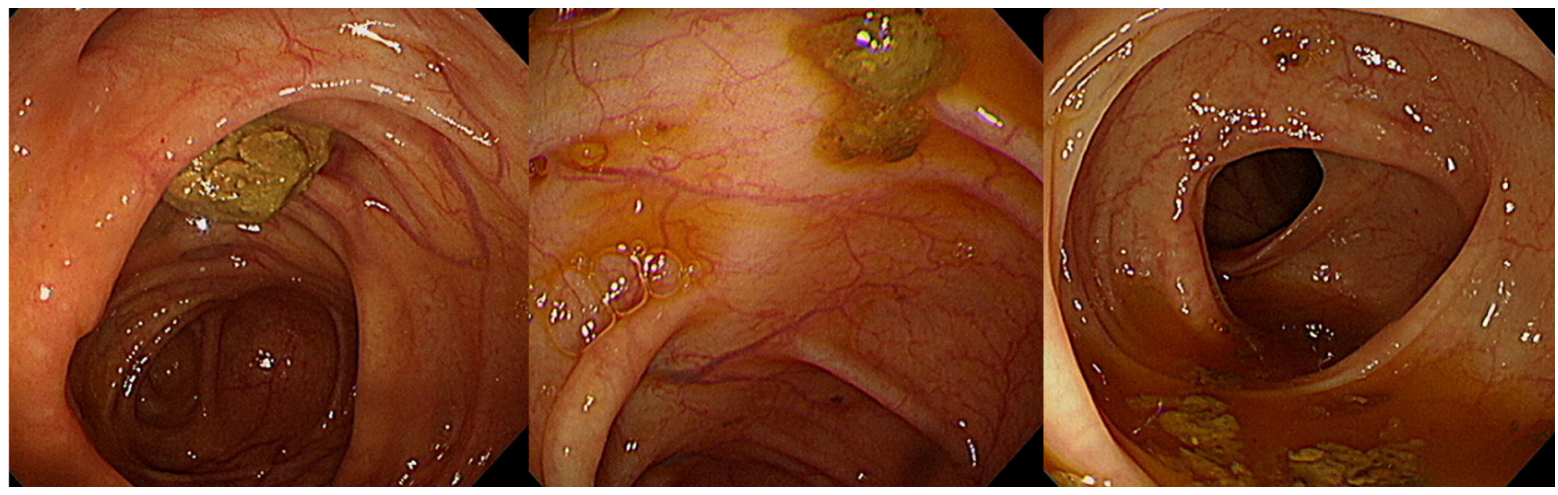

OSS (adequate)

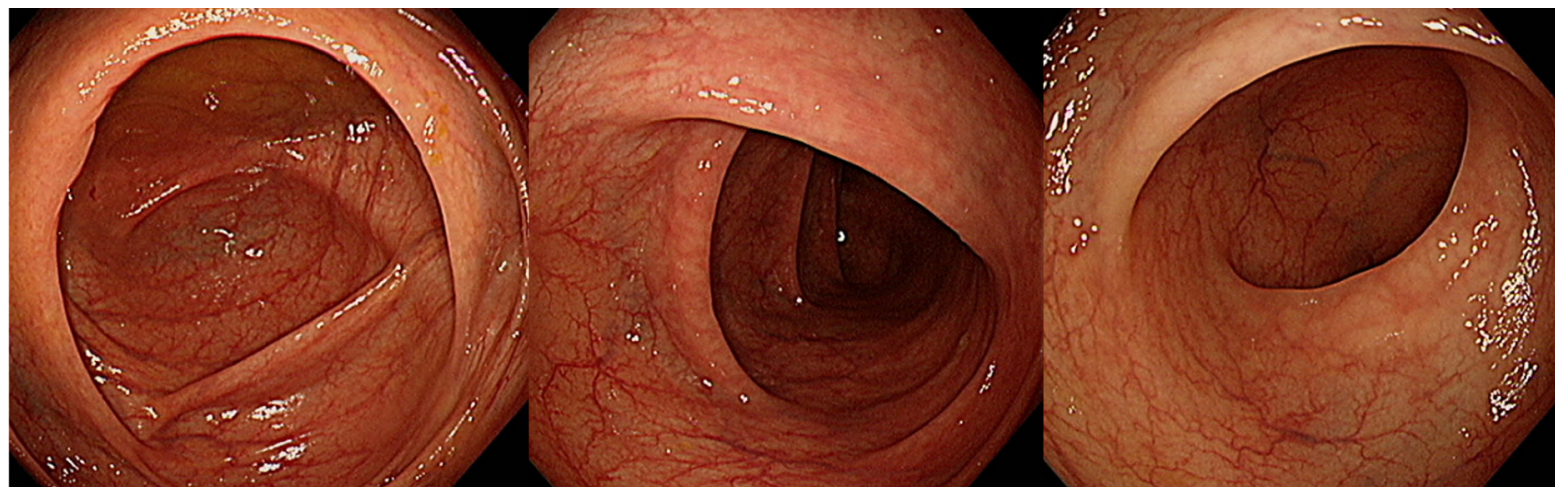

PEG-AA (inadequate)

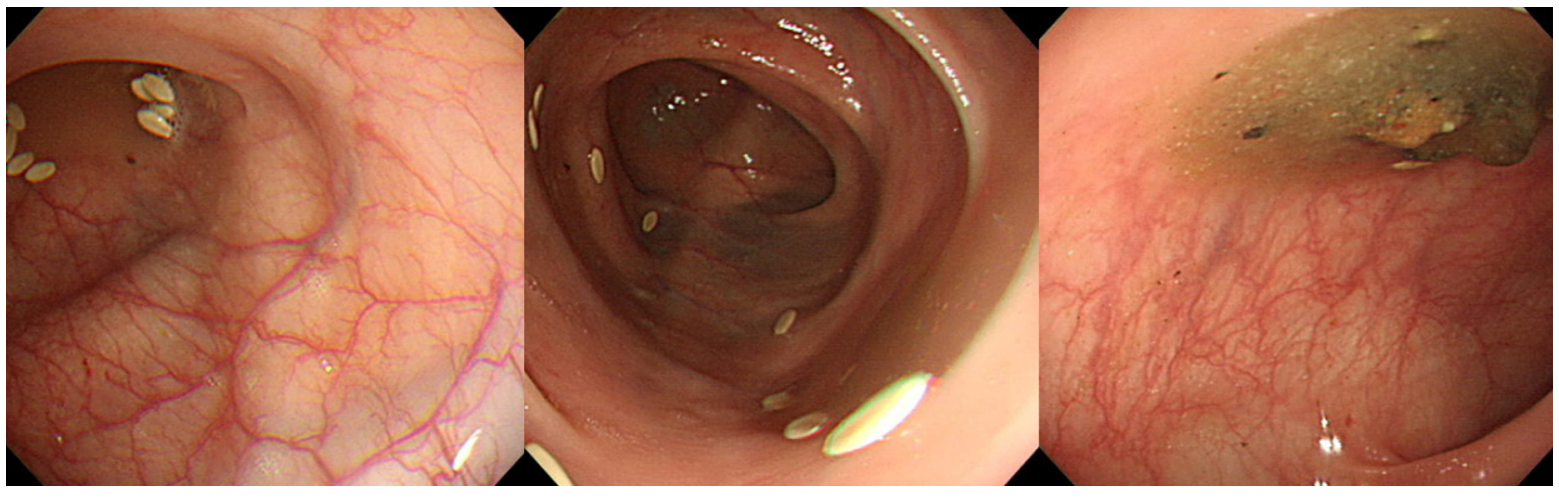

PEG-AA (adequate)

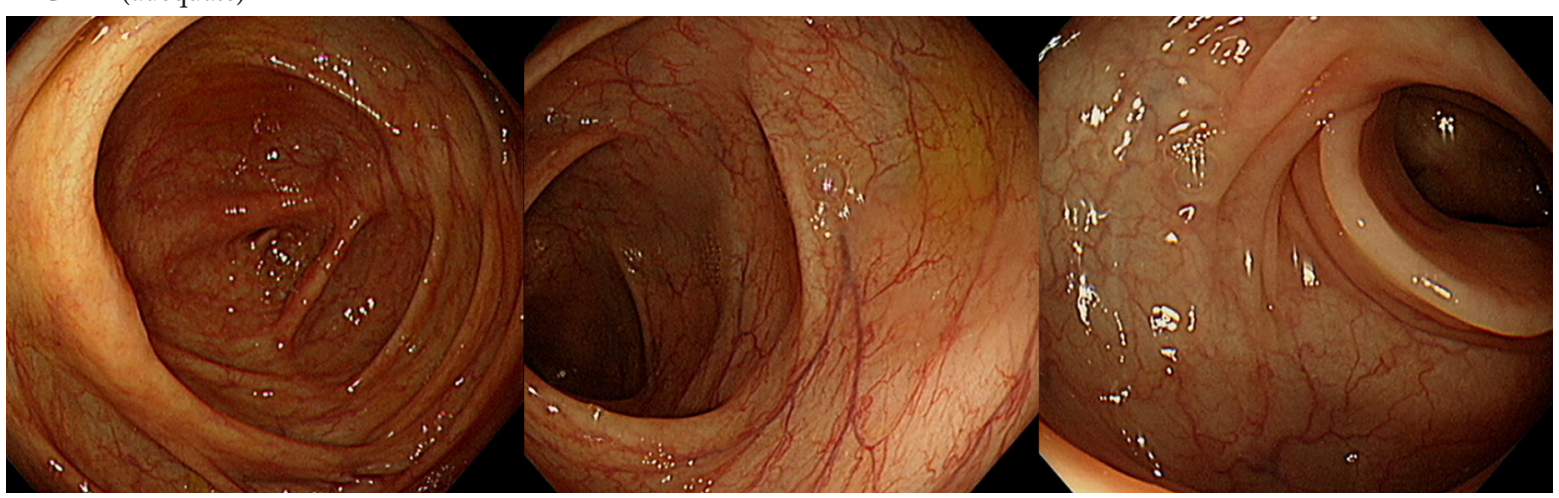

Fig. 1. Examples of unprepared and prepared colon segments (right, transverse, and left) in bowel cleansing with oral sulfate solution (OSS) and polyethylene glycol plus ascorbic acid (PEG-AA). 


\section{Statistical analyses}

The main outcome was quality of bowel preparation (inadequate vs. adequate). Independent sample $t$-tests or chisquared tests were used to compare the baseline and clinical characteristics between the patients receiving OSS and PEGAA. To determine the factors affecting the quality of the bowel preparations, logistic regression analyses were performed to determine adjusted odds ratios (ORs) and 95\% confidence intervals (CIs). The multivariate regression model included age, sex, hospitalization (inpatient/outpatient), timing of the examination (morning/afternoon), and the bowel cleansing formulation (OSS vs. PEG-AA). In addition, we performed subgroup analyses to determine whether the efficacy of the formulations differed according to patient characteristics. All two-sided $p$-values less than 0.05 were considered statistically significant. SPSS Statistics 19.0 (IBM, Armonk, NY, USA) was used for computation of statistical analyses.

\section{RESULTS}

\section{Baseline and clinical characteristics}

Overall, 167 patients (51.5\% male patients; mean age,
$55.3 \pm 14.2$ years of age, range, $17-82)$ were included in the study. The mean cecal intubation time (CIT) was $6.7 \pm 5.3$ min and the ADR was 29.3\%. In total, 106 (63.5\%) patients received OSS and 61 (36.5\%) patients received PEG-AA (Table 2). The mean age was different between the OSS group and the PEG-AA group (53.1 years of age vs. 59.1 years of age, $p=0.008)$. There were significantly more inpatients in the PEG-AA group than in the OSS group (32.8\% vs. $13.2 \%$, $p=0.002)$. Patients prepared by a split-dose accounted for $14.8 \%(9 / 61)$ of the patients in the PEG-AA group, whereas OSS was administered only as a split-dose. The mean CIT was longer in the PEG-AA group than in the OSS group (9.8 min vs. $4.9 \mathrm{~min}, p<0.001)$. Colonoscopy timing and ADR incidence were not different between the two groups.

The mean sum of the scores for BBPS (0-9) was 7.0 \pm 2.1 , with $2.1 \pm 0.9$ in the right colon, $2.5 \pm 0.7$ in the transverse colon, and $2.4 \pm 0.7$ in the left colon. The rate of inadequate bowel preparation was $19.8 \%$ (33/167): $12.3 \%$ in the OSS group and $32.8 \%$ in the PEG-AA group $(p=0.001)$. The mean BBPS scores between the OSS group and PEG-AA group were significantly different for all colon sections: total (7.5 vs. 6.1, $p<0.001$ ), right (2.3 vs. $1.8, p<0.001$ ), transverse (2.7 vs. 2.2 , $p=0.001$ ), and left (2.6 vs. 2.0, respectively, $p<0.001$ ).

Table 2. Baseline and Clinical Characteristics of the Patients

\begin{tabular}{lcccc}
\hline & Total $(\boldsymbol{n}=\mathbf{1 6 7})$ & OSS $(\boldsymbol{n = 1 0 6})$ & PEG-AA $(\boldsymbol{n}=\mathbf{6 1})$ & $\boldsymbol{p}$-value \\
\hline Age $(\mathrm{yr})$, mean \pm SD & $55.3 \pm 14.2$ & $53.1 \pm 13.8$ & $59.1 \pm 14.0$ & 0.008 \\
Male, $n(\%)$ & $86(51.5)$ & $58(54.7)$ & $28(45.9)$ & 0.272 \\
Hospitalization, $n(\%)$ & $34(20.4)$ & $14(13.2)$ & $20(32.8)$ & 0.002 \\
Morning colonoscopy, $n(\%)$ & $25(15.0)$ & $16(15.1)$ & $9(14.8)$ & 0.953 \\
Split-dose, $n(\%)$ & $115(68.9)$ & $106(100)$ & $9(14.8)$ & $<0.001$ \\
Inadequate bowel preparation & $33(19.8)$ & $13(12.3)$ & $20(32.8)$ & 0.001 \\
Cecal intubation time (min), mean \pm SD & $6.7 \pm 5.3$ & $4.9 \pm 3.9$ & $9.8 \pm 6.0$ & $<0.001$ \\
Adenoma detection rate, $n(\%)$ & $49(29.3)$ & $30(28.3)$ & $19(31.1)$ & 0.697 \\
\hline
\end{tabular}

OSS, oral sulfate solution; PEG-AA, polyethylene glycol plus ascorbic acid; SD, standard deviation.

Table 3. Risk Factors for Inadequate Bowel Preparation

\begin{tabular}{lcccc}
\hline Variables & Crude OR $(\mathbf{9 5 \%}$ CI) & p-value & Adjusted OR (95\% CI) & $p$-value \\
\hline Age & $1.01(0.98-1.04)$ & 0.403 & $0.99(0.97-1.03)$ & 0.842 \\
Male sex & $1.59(0.73-3.44)$ & 0.245 & $2.22(0.95-5.21)$ & 0.067 \\
OSS (vs. PEG-AA) & $0.29(0.13-0.63)$ & 0.002 & $0.26(0.11-0.63)$ & 0.003 \\
Morning (vs. afternoon) & $0.14(0.19-1.10)$ & 0.062 & $0.11(0.01-0.89)$ & 0.038 \\
Inpatient (vs. outpatient) & $1.64(0.68-3.95)$ & 0.274 & $1.36(0.49-3.74)$ & 0.558 \\
\hline
\end{tabular}

CI, confidence interval; OR, odds ratio; OSS, oral sulfate solution; PEG-AA, polyethylene glycol plus ascorbic acid. 
Table 4. Odds Ratios for Efficacy of Oral Sulfate Solution vs. Polyethylene Glycol Plus Ascorbic Acid in the Subgroups

\begin{tabular}{|c|c|c|c|c|c|c|}
\hline \multirow[t]{2}{*}{ Variables } & \multirow[t]{2}{*}{ Subgroups } & \multirow[t]{2}{*}{$n$} & \multicolumn{2}{|c|}{ Inadequate preparation, $n(\%)$} & \multirow[t]{2}{*}{ OR $(95 \% \mathrm{CI})$} & \multirow[t]{2}{*}{$p$-value } \\
\hline & & & OSS & PEG-AA & & \\
\hline \multirow[t]{2}{*}{ Age } & $<50 \mathrm{yr}$ & 57 & $7 / 40(17.5)$ & 2/17 (11.8) & $0.96(0.16-5.70)$ & 0.959 \\
\hline & $\geq 50 \mathrm{yr}$ & 110 & $6 / 66(9.1)$ & $18 / 44(40.9)$ & $0.13(0.04-0.41)$ & 0.001 \\
\hline \multirow[t]{2}{*}{ Sex } & Male & 86 & $11 / 58(19.0)$ & $9 / 28(32.1)$ & $0.58(0.19-1.79)$ & 0.339 \\
\hline & Female & 81 & $2 / 48(4.2)$ & $11 / 33(33.3)$ & $0.06(0.01-0.34)$ & 0.002 \\
\hline \multirow[t]{2}{*}{ Colonoscopy timing } & Morning & 25 & $0 / 16(0)$ & $1 / 9(11.1)$ & N/A & \\
\hline & Afternoon & 142 & $13 / 90(14.4)$ & $19 / 52(36.5)$ & $0.28(0.11-0.70)$ & 0.006 \\
\hline \multirow[t]{2}{*}{ Hospitalization } & Outpatient & 133 & $12 / 92(13.0)$ & $12 / 41(29.3)$ & $0.30(0.11-0.77)$ & 0.013 \\
\hline & Inpatient & 34 & $1 / 14(7.1)$ & $8 / 20(40.0)$ & $0.14(0.01-1.97)$ & 0.145 \\
\hline
\end{tabular}

Other covariates (age, sex, timing, hospitalization) were adjusted for all regressions.

CI, confidence interval; N/A, not available; OR, odds ratio; OSS, oral sulfate solution; PEG-AA, polyethylene glycol plus ascorbic acid.

\section{Factors related to bowel preparation quality}

The factors that affected the quality of bowel cleansing are presented in Table 3. In multivariate logistic regression, the OSS regimen was negatively associated with inadequate bowel preparation compared with PEG-AA (OR $=0.26$; 95\% CI, $0.11-0.63 ; p=0.003)$. Morning colonoscopies showed significantly better quality bowel preparation than afternoon examinations ( $\mathrm{OR}=0.11 ; 95 \% \mathrm{CI}, 0.01-0.89 ; p=0.038)$. Age, sex, and hospitalization did not affect the quality of bowel preparation. In the subgroup analyses, the OR for the efficacy of OSS vs. PEG-AA was 0.13 in patients of 50 years of age or older $(p=0.001)$ and 0.96 in patients younger than 50 years of age $(p=0.959)$ (Table 4$)$. In addition, good OSS efficacy was more prominent in females than in males $(\mathrm{OR}=0.06 ; p=0.002$ and $\mathrm{OR}=0.58 ; p=0.339$, respectively). There was also a significant difference between outpatients $(\mathrm{OR}=0.30 ; p=0.013)$ and inpatients $(\mathrm{OR}=0.14 ; p=0.145)$.

\section{DISCUSSION}

OSS was significantly more efficient for bowel preparation than PEG-AA, especially for older patients and female patients. We used the BBPS to assess the bowel cleansing quality for each colon section. The OSS group showed better preparation quality compared to the PEG-AA group, regardless of the colon section. In a prospective study, the inadequate preparation group on the Aronchick scale ${ }^{17}$ had a significantly higher rate of missed adenomas compared with the excellent preparation group $(\mathrm{OR}=3.04)$, whereas the $\mathrm{ADR}$ in the good or fair preparation groups did not differ from the excellent group. ${ }^{6}$ In other words, "inadequate or not" is appropriate as a criterion for the quality of bowel cleansing. Meanwhile, as OSS was approved for a split-dose,${ }^{15}$ OSS was prescribed only as a split-dose. Single-dose OSS has been associated with a higher incidence of gastrointestinal events, such as vomiting, compared with PEG-AA. ${ }^{15}$ Therefore, the preparation timing (split-dose vs. single-dose) could not be assessed by our retrospective design.

The pharmacological effect of the preparation formulation itself, as well as the tolerability and compliance related to its dose, taste, and preparation timing, can affect the quality of the bowel preparation. In this study, the cleansing efficacy between OSS and PEG-AA was different in patients of 50 years of age or older, but was similar in those younger than 50 years of age. Similarly, the difference was only found in female patients, not in male patients. These results suggested that older patients and female patients are vulnerable to the relatively large volume of 2 L PEG compared with OSS and that this led to a difference in compliance between the two regimens. Consistent with our results, a recent Korean study reported that split-dose OSS was more acceptable to elderly patients than PEG. ${ }^{18}$ Hospitalization influenced the efficacy of OSS vs. PEGAA in the subgroup analysis; however, it was limited because the inpatient sample size was insufficient. Further large-scale studies are required to confirm the results. In addition, as various comorbidities, besides inpatient status, can affect the quality of bowel preparation, ${ }^{19,20}$ detailed information on individual comorbidities may be helpful for the selection of preparation regimens based on safety and tolerability.

Morning examinations were more efficient than afternoon examinations in this study, irrespective of the bowel preparation regimen. The result was plausible because the intervals between the preparation and examination were shorter in the 
morning colonoscopies than in the afternoon examinations in both preparation regimens. The timing of preparation may be important for the quality of bowel cleansing. Published guidelines recommend an interval of no more than $4 \mathrm{~h}$ from the final dose of bowel preparation formulation to the examination. ${ }^{10}$ Previous studies showed that the preparation quality was significantly better in patients with a shorter interval between the bowel preparation and the start of the colonoscopy. ${ }^{21,22}$ Other results showing that split-dose preparation was superior to day-before cleansing may have been caused by different time intervals between bowel cleansing and the examination. ${ }^{23-25}$ In this study, it is possible that as all OSS regimens were prepared in a split-dose, this provided higher cleaning quality than PEG-AA, which was mainly prepared as a single-dose. The interval between the last dose of bowel preparation and the start time of colonoscopy would be helpful for the comparison. However, the individual data for the intervals were not available in our retrospective design.

Our study had several limitations. First, as this study was conducted in a single-center hospital, selection bias could not be excluded. Second, patient compliance with the preparation instructions and diet education can influence the quality of bowel cleansing. Even though we routinely provided direct education about the preparation methods to all patients scheduled for a colonoscopy, patient acceptance differs from person to person. In addition, high-residue diet, constipation, and high body mass index may be risk factors for inadequate bowel preparation ${ }^{8,22,26,27}$ and possible confounders. Third, the choice of formulation is inevitably affected by the physician's preference and the patient's medical status. Patients' underlying diseases may also affect the choice of preparation regimen and the cleaning quality of the regimen. Fourth, as we could not achieve a concordance among the physicians for the assessment of bowel preparations, the physician's subjectivity may have influenced the quality assessment. However, this issue may have been mitigated by our use of a verified scoring system for bowel cleansing. Finally, the OSS regimen was all split-dose, whereas the PEG-AA was mostly single-dose. Thus, the scope for comparison may be limited. Further studies are needed to compare the two regimens when each are provided as split-dose.

This paper focused on the effectiveness of the formulation, not the safety. This study supports previous studies ${ }^{13-15,28}$ that showed the non-inferiority of OSS compared with PEG-AA. Interestingly, OSS may be of benefit for older patients and female patients. A strength of this study was that the comparison of the preparation efficacy between the two regimens was stratified by patient characteristics, which indicated that the bowel cleansing regimen should be selected with consideration of the patient characteristics and their subsequent compliance.
Conflicts of Interest

The authors have no financial conflicts of interest.

Author Contributions

Conceptualization: Yun Jeong Lim

Data curation: Seok Bo Hong, Seongju Lee

Formal analysis: Ji Hyung Nam, SBH

Methodology: YJL

Writing-original draft: JHN, SBH

Writing-review\&editing: Hyoun Woo Kang, Jae Hak Kim, Jin Ho Lee

ORCID

Ji Hyung Nam: https://orcid.org/0000-0002-7083-7581

Seok Bo Hong: https://orcid.org/0000-0002-1246-9071

Seongju Lee: https://orcid.org/0000-0002-3727-1731

Hyoun Woo Kang: https://orcid.org/0000-0003-3431-0827

Jae Hak Kim: https://orcid.org/0000-0001-6270-3703

Jin Ho Lee: https://orcid.org/0000-0002-8013-3262

\section{REFERENCES}

1. Inadomi JM. Screening for colorectal neoplasia. N Engl J Med 2017;376:149-156.

2. Johnson DA, Barkun AN, Cohen LB, et al. Optimizing adequacy of bowel cleansing for colonoscopy: recommendations from the US multi-society task force on colorectal cancer. Gastroenterology 2014;147:903-924.

3. ASGE Standards of Practice Committee, Saltzman JR, Cash BD, et al. Bowel preparation before colonoscopy. Gastrointest Endosc 2015;81:781-794.

4. Solad Y, Wang C, Laine L, et al. Influence of colonoscopy quality measures on patients' colonoscopist selection. Am J Gastroenterol 2015;110:215-219.

5. Rex DK, Schoenfeld PS, Cohen J, et al. Quality indicators for colonoscopy. Gastrointest Endosc 2015;81:31-53.

6. Hong SN, Sung IK, Kim JH, et al. The effect of the bowel preparation status on the risk of missing polyp and adenoma during screening colonoscopy: a tandem colonoscopic study. Clin Endosc 2012;45:404-411.

7. Lai EJ, Calderwood AH, Doros G, Fix OK, Jacobson BC. The Boston bowel preparation scale: a valid and reliable instrument for colonoscopy-oriented research. Gastrointest Endosc 2009;69(3 Pt 2):620-625.

8. Rotondano G, Rispo A, Bottiglieri ME, et al. Quality of bowel cleansing in hospitalized patients undergoing colonoscopy: a multicentre prospective regional study. Dig Liver Dis 2015;47:669-674.

9. Rex DK, Imperiale TF, Latinovich DR, Bratcher LL. Impact of bowel preparation on efficiency and cost of colonoscopy. Am J Gastroenterol 2002;97:1696-1700.

10. Hassan C, Bretthauer M, Kaminski MF, et al. Bowel preparation for colonoscopy: European Society of Gastrointestinal Endoscopy (ESGE) guideline. Endoscopy 2013;45:142-150.

11. Xie Q, Chen L, Zhao F, et al. A meta-analysis of randomized controlled trials of low-volume polyethylene glycol plus ascorbic acid versus standard-volume polyethylene glycol solution as bowel preparations for colonoscopy. PLoS One 2014;9:e99092.

12. Moon CM, Park DI, Choe YG, et al. Randomized trial of 2-L polyethylene glycol + ascorbic acid versus 4-L polyethylene glycol as bowel cleansing for colonoscopy in an optimal setting. J Gastroenterol Hepatol 2014;29:1223-1228.

13. Tajika M, Tanaka T, Ishihara M, et al. A randomized controlled trial evaluating a low-volume PEG solution plus ascorbic acid versus standard PEG solution in bowel preparation for colonoscopy. Gastroenterol Res Pract 2015;2015:326581. 
14. Kim B, Lee SD, Han KS, et al. Comparative evaluation of the efficacy of polyethylene glycol with ascorbic acid and an oral sulfate solution in a split method for bowel preparation: a randomized, multicenter phase III clinical trial. Dis Colon Rectum 2017;60:426-432.

15. Di Palma JA, Rodriguez R, McGowan J, Cleveland M. A randomized clinical study evaluating the safety and efficacy of a new, reduced-volume, oral sulfate colon-cleansing preparation for colonoscopy. Am J Gastroenterol 2009;104:2275-2284.

16. Rex DK, Di Palma JA, Rodriguez R, McGowan J, Cleveland M. A randomized clinical study comparing reduced-volume oral sulfate solution with standard 4-liter sulfate-free electrolyte lavage solution as preparation for colonoscopy. Gastrointest Endosc 2010;72:328-336.

17. Kastenberg D, Bertiger G, Brogadir S. Bowel preparation quality scales for colonoscopy. World J Gastroenterol 2018;24:2833-2843.

18. Kwak MS, Cha JM, Yang HJ, et al. Safety and efficacy of low-volume preparation in the elderly: oral sulfate solution on the day before and split-dose regimens (SEE SAFE) study. Gut Liver 2019;13:176-182.

19. Sim JS, Koo JS. Predictors of inadequate bowel preparation and salvage options on colonoscopy. Clin Endosc 2016;49:346-349.

20. Hassan C, Fuccio L, Bruno M, et al. A predictive model identifies patients most likely to have inadequate bowel preparation for colonoscopy. Clin Gastroenterol Hepatol 2012;10:501-506.

21. Longcroft-Wheaton G, Bhandari P. Same-day bowel cleansing regimen is superior to a split-dose regimen over 2 days for afternoon colo- noscopy: results from a large prospective series. J Clin Gastroenterol 2012;46:57-61.

22. Eun CS, Han DS, Hyun YS, et al. The timing of bowel preparation is more important than the timing of colonoscopy in determining the quality of bowel cleansing. Dig Dis Sci 2011;56:539-544.

23. Martel M, Barkun AN, Menard C, Restellini S, Kherad O, Vanasse A. Split-dose preparations are superior to day-before bowel cleansing regimens: a meta-analysis. Gastroenterology 2015;149:79-88.

24. Kilgore TW, Abdinoor AA, Szary NM, et al. Bowel preparation with split-dose polyethylene glycol before colonoscopy: a meta-analysis of randomized controlled trials. Gastrointest Endosc 2011;73:1240-1245.

25. Radaelli F, Paggi S, Repici A, et al. Barriers against split-dose bowel preparation for colonoscopy. Gut 2017;66:1428-1433.

26. Wu KL, Rayner CK, Chuah SK, Chiu KW, Lu CC, Chiu YC. Impact of low-residue diet on bowel preparation for colonoscopy. Dis Colon Rectum 2011;54:107-112.

27. Soweid AM, Kobeissy AA, Jamali FR, et al. A randomized single-blind trial of standard diet versus fiber-free diet with polyethylene glycol electrolyte solution for colonoscopy preparation. Endoscopy 2010;42:633638.

28. Lee HH, Lim CH, Kim JS, et al. Comparison between an oral sulfate solution and a $2 \mathrm{~L}$ of polyethylene glycol/ascorbic acid as a split dose bowel preparation for colonoscopy. J Clin Gastroenterol 2019;53:e431-e437. 